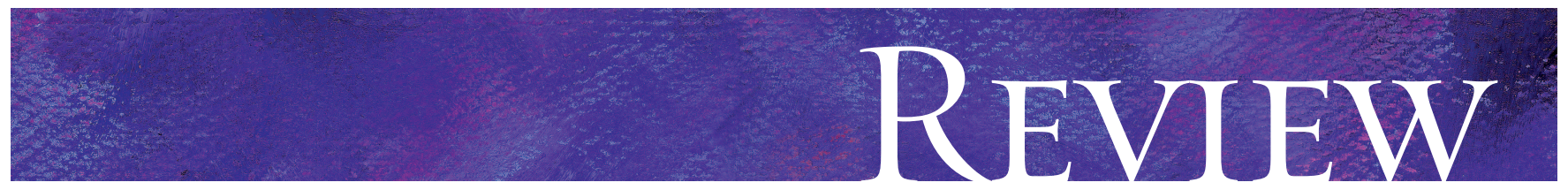

\title{
Prescribing exercise as preventive therapy
}

\author{
Darren E.R. Warburton, Crystal Whitney Nicol, Shannon S.D. Bredin
}

\section{ABSTRACT}

Energy expenditure of about $1000 \mathrm{kcal}(4200 \mathrm{~kJ})$ per week (equivalent to walking 1 hour 5 days a week) is associated with significant health benefits. Health benefits can be achieved through structured or nonstructured physical activity, accumulated throughout the day (even through short 10-minute bouts) on most days of the week. In this article we outline the means of evaluating cardiovascular and musculoskeletal fitness, the methods of evaluating physical activity levels, the current recommendations for exercise (including intensity, type, time and frequency) and the resources available for patients and physicians interested in learning more about the evaluation of physical activity and fitness levels and the prescription of exercise.

CMAJ 2006;174(7):96I-74

$\mathrm{T}$ here is incontrovertible evidence from observational and randomized trials that regular physical activity contributes to the primary and secondary prevention of cardiovascular disease and several other chronic conditions and that it is associated with a reduced risk of premature death. ${ }^{1}$ Physical activity can be recommended as a preventive therapy to people of all ages.

Physical fitness refers to a physiologic state of well-being that allows one to meet the demands of daily living (healthrelated physical fitness) or that provides the basis for sport performance (performance-related physical fitness), or both. Health-related physical fitness involves the components of physical fitness related to health status, including cardiovascular fitness, musculoskeletal fitness, body composition and metabolism. There are numerous ways to assess a person's general health-related physical fitness. For example, the primary assessment tool in Canada - the Canadian Physical Activity, Fitness and Lifestyle Approach ${ }^{2}$

o (Box I) - provides a variety of testing options, from a full of appraisal to an information session (providing help, tips and advice only). Health and fitness professionals certified to conduct these measures can be found throughout Canada and can be located through the Health and Fitness Program of the Canadian Society for Exercise Physiology (www.csep .ca/hfp.asp).

\section{Methods of evaluating health-related physical fitness}

Physical fitness can be easily assessed with the use of wellestablished appraisal protocols from agencies such as the American College of Sports Medicine ${ }^{3}$ and the Canadian Society for Exercise Physiology. ${ }^{4}$ These assessments are designed to evaluate the individual components of health-related physical fitness, including body composition, aerobic fitness and musculoskeletal fitness (muscular strength, muscular endurance, power and flexibility).

The terms "aerobic" and "anaerobic" are commonly used to define fitness in the health and fitness industry. However, many are not fully aware of the distinction between these fitness designations. In brief, aerobic fitness refers to the body's ability to transport and use oxygen during prolonged strenuous exercise or work. ${ }^{5,6}$ Anaerobic fitness refers to the body's ability to produce energy without the use of oxygen.

Maximum anaerobic power (the maximum rate at which energy is produced without the use of oxygen) is generally

Box 1: Components of the Canadian Physical Activity, Fitness and Lifestyle Approach, ${ }^{2}$ a tool used to assess health-related physical fitness

- The Healthy Physical Activity Questionnaire, to assess current levels of physical activity

- The Healthy Lifestyle Questionnaire, to evaluate current lifestyle habits (e.g., nutrition, alcohol consumption)

- A pre-activity health screening tool (i.e., PAR-Q), to identify people for whom certain physical activities may be inappropriate and those who should seek medical advice (e.g., people with established cardiovascular disease)

- Measurement of resting blood pressure and heart rate

- Assessment of body composition (body mass index, waist circumference and skinfold thickness [a measure of body fat])

- Canadian Aerobic Fitness Test, to assess aerobic fitness. (Changes to the assessment tool are underway to include other measures of aerobic fitness, such as the Rockport One Mile Walk Test, and submaximal treadmill and cycle tests)

- Assessment of musculoskeletal fitness (grip strength, push-ups, sit-and-reach test, partial curl-ups, vertical jump and back-extension endurance) and back health 
taken as the standard measure of anaerobic fitness. There are numerous means to directly and indirectly assess people's maximum anaerobic power, including their all-out efforts while cycling, running or jumping. Most health professionals do not assess maximum anaerobic power owing to the difficulty that different patient populations would have in performing many of these tests (in particular elderly patients). However, recent researchers have postulated that anaerobic capacity plays an important role in the performance of many activities of daily living. ${ }^{5,6}$ This has led some, including our own group, to advocate the inclusion of the assessment of anaerobic fitness in the evaluation of health status. ${ }^{7}$

Aerobic fitness is commonly measured by a person's maximum aerobic power $\left(\mathrm{VO}_{2 \max }\right)$, the maximum amount of oxygen that can be transported and used by the working muscles. The direct assessment of $\mathrm{VO}_{2 \max }$ in a laboratory setting is generally conducted with the use of commercially available metabolic carts and requires highly trained staff. Owing to the complexity and cost of the direct assessment, many health and fitness professionals prefer to estimate $\mathrm{VO}_{2 \max }$ without measuring oxygen consumption.

A variety of tests are available to measure aerobic fitness indirectly, including submaximal tests (e.g., the Rockport One Mile Test, the modified Canadian Aerobic Fitness Test and the YMCA cycle ergometer protocol) and incremental to maximal tests (e.g., the Bruce protocol) that involve a variety of exercise modalities (e.g., cycling, running, stair climbing, rowing). Often heart rate is used to estimate $\mathrm{VO}_{2 \max }$ during submaximal or maximal exercise tests. A lower heart rate for a given workload is thought to represent a higher level of aerobic fitness. Many fitness and health professionals prefer to use exercise time or estimated oxygen cost (e.g., metabolic equivalents [METs]) for the last stage completed during an incremental protocol to estimate aerobic fitness. To achieve a reasonable and reliable estimate of $\mathrm{VO}_{2 \max }$, these indirect assessments must be conducted in a highly standardized and reproducible fashion.

Musculoskeletal fitness can be assessed relatively easily within and outside of the laboratory setting. Common tests include grip strength (muscular strength), push-ups (muscular endurance and strength), curl-ups (muscular endurance) and sit-and-reach tests (flexibility). These tests can be performed safely and in a reproducible fashion by people of all ages. Although more sophisticated procedures and equipment may be used, these simple tests are thought to be more than adequate for assessing a person's current level of healthrelated physical fitness.

For physicians and fitness professionals interested in assessing a patient's current health-related physical fitness, the tests outlined in the manual for the Canadian Physical Activity, Fitness and Lifestyle Approach (available for purchase online through the Canadian Society for Exercise Physiology, www.csep.ca/publications.asp) are relatively easy to administer. For instance, the Rockport One Mile Walk test and the modified Canadian Aerobic Fitness (step) Test, used to assess aerobic fitness, require little or no equipment. The Rockport test requires only a suitable walking surface (preferably a 400$\mathrm{m}$ track), a stop watch and the ability to monitor the patient's heart rate (e.g., through palpation). The step test requires steps of standard height (8 inches $[20.3 \mathrm{~cm}])$. For musculoskeletal fitness tests, little equipment is required (e.g., a mat for push-ups and curl-ups, and a standard ruler for the sit-and-reach test).

Health practitioners should be aware that there are subtle differences between patient groups with respect to fitness testing. For instance, the attainment of a "true" $\mathrm{VO}_{2 \max }$ in children is difficult in laboratory settings. Fortunately, a series of field tests have been developed (e.g., the Leger 20-m shuttle test ${ }^{8}$ ) that provide valid and reliable determinants of aerobic fitness. Also, it may be best to ask children to perform running activities instead of cycling activities because of their less developed muscular strength. ${ }^{9}$

Although fitness testing is important and arguably essential for elderly people, particular care must be taken when administering current popular fitness assessments. The American College of Sports Medicine has outlined special considerations that must be taken when assessing the physical fitness of elderly people. ${ }^{10}$ For example, elderly people are at increased risk of arrhythmias during exercise, and they commonly use medications that may affect physiologic responses to exercise. Also, they may require smaller initial workloads and smaller changes in workloads during testing than do other age groups. Furthermore, it is preferable to use equipment that promotes safety (e.g., treadmills with handrails, cycle ergometers). Because of the variability in maximum heart rate in elderly people, the direct assessment of maximum heart rate is often preferable for exercise prescription. ${ }^{9}$

For obese people, one must be aware of the effect of obesity on their ability to conduct certain tests and the physiologic response to exercise. For instance, obese people may be more comfortable using exercise equipment that supports their body mass. Therefore, a cycle ergometer may be preferable to a treadmill. Moreover, obese patients often do not tolerate running well; therefore, walking tests may be preferable to running protocols. Obese people may also be prone to orthopedic injuries, and their heart rate response to exercise may differ from that of nonobese people ${ }^{11}$ (obese people often have lower maximum heart rates).

Special care must also be taken during the assessment of of people with chronic disease. For instance, patients with cardiovascular disease should be monitored closely during physiologic testing. The appraiser must have a clear understanding of the effects of the patient's clinical status and medications on the physiologic response to exercise.

A simple preliminary tool for patient self-assessment of physical activity levels is provided in Appendix I. Patients may respond to their own assessments and be more willing to discuss improvements in their activity levels using this brief instrument. Such physical activity scales are often used in large population-based studies. They also provide a baseline estimate of a person's relative activity levels. However, the use of self-assessment questionnaires as an exercise prescription tool is limited compared with other, more objective methods (e.g., determining physical fitness and physiologic responses to exercise). As such, most health 
professionals use self-assessment questionnaires only as a means of evaluating current physical activity levels and instead rely on other methods to develop specific exercise prescriptions.

\section{Prescribing exercise: intensity, time, type and frequency}

Guidelines for improving physical activity and fitness have evolved continually as new evidence becomes available on the levels of exercise required for health benefits. In general, these guidelines can be separated into 4 main strategies (Box 2) that physicians and patients can use to develop their own exercise prescriptions. Some patients will be interested in pursuing all 4 strategies, whereas others may be more compliant if they follow one strategy. For example, low-intensity exercise is generally better accepted by people

Box 2: Recommended levels of exercise required to improve physical activity and fitness levels for health benefits

Low-intensity (light effort) aerobic exercise

- $20 \%-39 \%$ of heart rate reserve, or about 2-4 METs (metabolic equivalents)

- About 60 min per day

- Most (preferably all) days of the week

- Examples: light gardening, light walking

Moderate-intensity aerobic exercise

- $40 \%-59 \%$ of heart rate reserve, or about 4-6 METs

- 20-60 min per day

- 3-5 days per week

- Examples: brisk walking (15-20 min per mile), dancing

High-intensity aerobic exercise

- $60 \%-84 \%$ of heart rate reserve, or about 6-8 METs

- 20-60 min per day

- 3-5 days per week

- Examples: jogging, swimming

Resistance and flexibility exercise

- 1-2 sets (each set 8-12 repetitions) of 8-10 different resistance exercises of moderate intensity that engage the large muscle groups, 2-4 days per week

- People over $60 \mathrm{yr}$ and frail people may need to engage in more repetitions (10-15) to compensate for a lower resistance requirement

- Gentle reaching, bending and stretching exercises of the major muscle groups to improve flexibility (hold each stretch for 10-30 seconds) for a minimum of 2-3 days per week (preferably 4-7)

Note: Aerobic exercise can be accumulated in short (10-minute) sessions of activity throughout the day. The approximate MET values provided are estimates for middle-aged adults (40-64 yr). The required METs would be lower for elderly and very elderly people, and higher for younger adults. ${ }^{15}$ In general, the higher the intensity of activity, the less time required for health benefits. Each aerobic exercise session should begin with a warm-up (exercise designed to raise heart rate and body temperature) and end with a cool-down (mild exercise designed to slowly decrease heart rate and body temperature). naive to exercise training, those who are extremely deconditioned ("out of shape") and older people. Low-intensity exercise may result in an improvement in health status with little or no change in physical fitness (as outlined in the companion article ${ }^{1}$ ). In fact, light or moderate activity is associated with a reduced risk of death from any cause among men with established coronary artery disease. Furthermore, regular walking or moderate to heavy gardening has been shown to be sufficient in achieving these health benefits. ${ }^{12}$ Maintaining an active lifestyle or taking up light or moderate physical activity significantly reduces the risk of cardiovascular disease and death from any cause among older men (with or without established cardiovascular disease). ${ }^{13}$ This is important information given the preferred leisure activities of adults (walking, gardening and homebased exercise). ${ }^{14}$

The intensity of aerobic training can vary. For example, low-fit people can attain significant improvements in physical fitness with a lower training intensity (e.g., $40 \%-50 \%$ of heart rate reserve) than that needed by people with a higher baseline fitness level, whereas the latter would need a greater level of exercise intensity to achieve further improvements in fitness. ${ }^{15,16}$ Furthermore, extremely deconditioned people may improve physical fitness with as little as 2 exercise sessions per week. ${ }^{17}$ In fact, some have shown an improvement in aerobic fitness with exercise intensities as low as 30\% of heart rate reserve in sedentary people. ${ }^{18}$ Many (including our own research group) have recently advocated the inclusion of short bouts of high-intensity exercise. However, adherence to this form of exercise may be poor and the risk of musculoskeletal injury high, especially in people unaccustomed to exercise. ${ }^{19,20}$

\section{How much exercise should be recommended?}

Many health professionals recommend a minimum level of energy expenditure (volume of physical activity) of about I000 kcal (4200 kJ) per week, acknowledging the additive benefits of higher levels of energy expenditure. Expending I000 kcal (4200 kJ) per week is equivalent to I hour of moderate walking 5 days a week. However, as outlined in the companion paper, ${ }^{1}$ a lower level may also achieve health benefits. ${ }^{21}$ In fact, the American College of Sports Medicine has stated that health benefits occur with energy expenditures as low as $700 \mathrm{kcal}(2940 \mathrm{~kJ})$ per week, with additional benefits occurring at higher levels. ${ }^{15}$

The recommended daily energy expenditure for health is currently $150-400 \mathrm{kcal}(630-1680 \mathrm{~kJ})$ per day. ${ }^{9}$ For instance, if a previously sedentary person exercised at the lower end of the recommended amount ( $150 \mathrm{kcal}[630 \mathrm{~kJ}]$ ) on most (6) days of the week, he or she would approach the health-related goal of $1000 \mathrm{kcal}(4200 \mathrm{~kJ})$ per week. It is important to stress that an increase of $1000 \mathrm{kcal}(4200 \mathrm{~kJ})$ per week in physical activity or of I MET in physical fitness appears to confer a mortality benefit of $20 \%{ }^{22}$ This highlights the importance of commencing a training program that is progressive in nature. 
Many prefer to focus on the intensity of the activity. Here the nature of the activity and the speed with which it is accomplished provide estimates of energy expenditure per minute (often relative to body mass). Thus, prescriptive targets can be set in terms of energy output, as measured in kilocalories (kilojoules) per minute, METs or oxygen consump- tion $\left(\mathrm{VO}_{2}\right.$, millilitres of oxygen per kilogram per minute).

There are several means of determining the "dose" of exercise. In addition to the type of exercise, there are 3 modifiable components of an exercise prescription: intensity, duration and frequency. Table I provides the estimated times required to meet daily energy expenditures for various physi-

Table 1: Estimated time required to meet recommended daily energy expenditures for common activities*

\begin{tabular}{|c|c|c|c|c|c|c|c|c|c|c|c|}
\hline \multirow[b]{2}{*}{ Activity } & \multirow[b]{2}{*}{ METs } & \multirow[b]{2}{*}{$\mathrm{EE}$} & \multicolumn{9}{|c|}{ Body mass, $\mathrm{kg}$; time required to meet daily energy expenditure, min } \\
\hline & & & 50 & 60 & 70 & 80 & 90 & 100 & 110 & 120 & 130 \\
\hline \multicolumn{12}{|l|}{ Leisure } \\
\hline Backpacking & 7.0 & 0.12 & 26 & 21 & 18 & 16 & 14 & 13 & 12 & 11 & 10 \\
\hline Basketball, game & 8.0 & 0.13 & 23 & 19 & 16 & 14 & 13 & 11 & 10 & 9 & 9 \\
\hline Basketball, shooting baskets & 4.5 & 0.08 & 40 & 33 & 29 & 25 & 22 & 20 & 18 & 17 & 15 \\
\hline Bicycling, general stationary & 7.0 & 0.12 & 26 & 21 & 18 & 16 & 14 & 13 & 12 & 11 & 10 \\
\hline $\begin{array}{l}\text { Bicycling, light (10.0-11.9 mph } \\
[16-19.2 \mathrm{kph}])\end{array}$ & 6.0 & 0.10 & 30 & 25 & 21 & 19 & 17 & 15 & 14 & 13 & 12 \\
\hline $\begin{array}{l}\text { Bicycling, moderate }(12.0-13.9 \mathrm{mph} \\
[19.3-22.4 \mathrm{kph}])\end{array}$ & 8.0 & 0.13 & 23 & 19 & 16 & 14 & 13 & 11 & 10 & 9 & 9 \\
\hline $\begin{array}{l}\text { Bicycling, vigorous (14.0-15.9 mph } \\
\text { [22.5-25.6 kph]) }\end{array}$ & 10.0 & 0.17 & 18 & 15 & 13 & 11 & 10 & 9 & 8 & 8 & 7 \\
\hline Bowling & 3.0 & 0.05 & 60 & 50 & 43 & 38 & 33 & 30 & 27 & 25 & 23 \\
\hline $\begin{array}{l}\text { Conditioning exercise, calisthenics (light to } \\
\text { moderate) }\end{array}$ & 3.5 & 0.06 & 51 & 43 & 37 & 32 & 29 & 26 & 23 & 21 & 20 \\
\hline Conditioning exercise, calisthenics (vigorous) & 8.0 & 0.13 & 23 & 19 & 16 & 14 & 13 & 11 & 10 & 9 & 9 \\
\hline $\begin{array}{l}\text { Conditioning exercise, stair-treadmill } \\
\text { ergometer }\end{array}$ & 9.0 & 0.15 & 20 & 17 & 14 & 13 & 11 & 10 & 9 & 8 & 8 \\
\hline Curling & 4.0 & 0.07 & 45 & 38 & 32 & 28 & 25 & 23 & 20 & 19 & 17 \\
\hline Dancing, general aerobic & 6.5 & 0.11 & 28 & 23 & 20 & 17 & 15 & 14 & 13 & 12 & 11 \\
\hline Dancing, social or ballroom (fast) & 4.5 & 0.08 & 40 & 33 & 29 & 25 & 22 & 20 & 18 & 17 & 15 \\
\hline Fishing, from a boat (sitting) & 2.5 & 0.04 & 72 & 60 & 51 & 45 & 40 & 36 & 33 & 30 & 28 \\
\hline Fishing, in a stream (waders) & 6.0 & 0.10 & 30 & 25 & 21 & 19 & 17 & 15 & 14 & 13 & 12 \\
\hline Frisbee playing & 3.0 & 0.05 & 60 & 50 & 43 & 38 & 33 & 30 & 27 & 25 & 23 \\
\hline Golfing, using a powercart & 3.5 & 0.06 & 51 & 43 & 37 & 32 & 29 & 26 & 23 & 21 & 20 \\
\hline Golfing, walking and carrying clubs & 4.5 & 0.08 & 40 & 33 & 29 & 25 & 22 & 20 & 18 & 17 & 15 \\
\hline Golfing, walking and pulling clubs & 4.3 & 0.07 & 42 & 35 & 30 & 26 & 23 & 21 & 19 & 17 & 16 \\
\hline Hiking, cross-country & 6.0 & 0.10 & 30 & 25 & 21 & 19 & 17 & 15 & 14 & 13 & 12 \\
\hline Ice hockey & 8.0 & 0.13 & 23 & 19 & 16 & 14 & 13 & 11 & 10 & 9 & 9 \\
\hline Jogging, general & 7.0 & 0.12 & 26 & 21 & 18 & 16 & 14 & 13 & 12 & 11 & 10 \\
\hline Playing catch, football or baseball & 2.5 & 0.04 & 72 & 60 & 51 & 45 & 40 & 36 & 33 & 30 & 28 \\
\hline Rollerblading, in-line skating & 12.5 & 0.21 & 14 & 12 & 10 & 9 & 8 & 7 & 7 & 6 & 6 \\
\hline $\begin{array}{l}\text { Running, } 5.0 \mathrm{mph}(8 \mathrm{kph}) ; 12 \mathrm{~min} / \mathrm{mile} \\
(7.5 \mathrm{~min} / \mathrm{km})\end{array}$ & 8.0 & 0.13 & 23 & 19 & 16 & 14 & 13 & 11 & 10 & 9 & 9 \\
\hline $\begin{array}{l}\text { Running, } 7.5 \mathrm{mph}(12 \mathrm{kph}) ; 8 \mathrm{~min} / \mathrm{mile} \\
(5 \mathrm{~min} / \mathrm{km})\end{array}$ & 12.5 & 0.21 & 14 & 12 & 10 & 9 & 8 & 7 & 7 & 6 & 6 \\
\hline $\begin{array}{l}\text { Running, } 10.9 \mathrm{mph}(17.5 \mathrm{kph}) ; 5.5 \mathrm{~min} / \mathrm{mile} \\
(3.4 \mathrm{~min} / \mathrm{km})\end{array}$ & 18.0 & 0.30 & 10 & 8 & 7 & 6 & 6 & 5 & 5 & 4 & 4 \\
\hline $\begin{array}{l}\text { Skiing, cross-country, } 4.0-4.9 \mathrm{mph} \\
\text { (6.4-7.9 kph) (moderate) }\end{array}$ & 8.0 & 0.13 & 23 & 19 & 16 & 14 & 13 & 11 & 10 & 9 & 9 \\
\hline Skiing, water & 6.0 & 0.10 & 30 & 25 & 21 & 19 & 17 & 15 & 14 & 13 & 12 \\
\hline Skiing, downhill & 6.0 & 0.10 & 30 & 25 & 21 & 19 & 17 & 15 & 14 & 13 & 12 \\
\hline Snowmobiling & 3.5 & 0.06 & 51 & 43 & 37 & 32 & 29 & 26 & 23 & 21 & 20 \\
\hline Snowshoeing & 8.0 & 0.13 & 23 & 19 & 16 & 14 & 13 & 11 & 10 & 9 & 9 \\
\hline
\end{tabular}


cal activities (leisure and daily living) according to different body weights. Table 2 shows the estimated energy expenditures that a $70-\mathrm{kg}$ person would achieve while performing various physical activities for different durations. Using these tables, it is possible for a person to estimate his or her energy expenditure and the absolute intensity of exercise.
Box 3 contains examples of individualized exercise prescriptions for patients who are interested in increasing their physical activity levels. They are based on the standardized activity and energy expenditure values in Table I and Table 2. Because these tables provide only estimates of the energy expenditure associated with various forms of activities, exer-

Table 1 continued

Body mass, $\mathrm{kg}$; time required to meet daily energy expenditure, min

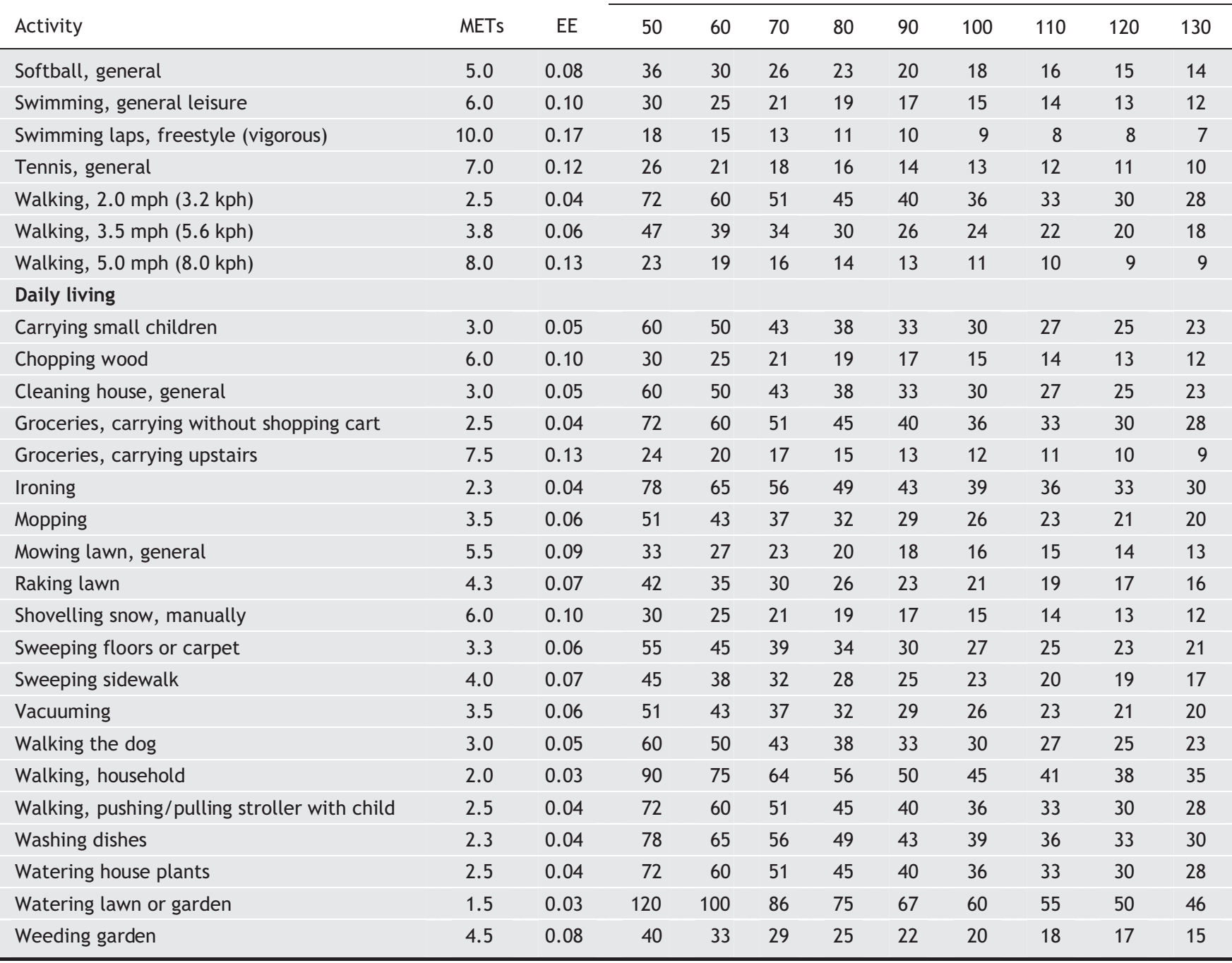

Note: $M E T s=$ metabolic equivalents $(1 \mathrm{MET}=3.5 \mathrm{~mL}$ oxygen per kilogram per minute, or $1 \mathrm{kcal}$ [4.2 $\mathrm{kJ}$ ] per kilogram per hour $)$, EE = energy expenditure (kilocalories) per kilogram per minute.

${ }^{*}$ Data were derived from the Compendium of Physical Activities. ${ }^{23,24}$ The latest version of this compendium ${ }^{23}$ contains absolute energy expenditures associated with 605 different physical activities, including activities of daily living.

Guidelines to using this chart

- A minimum energy expenditure of $150-400 \mathrm{kcal}$ (630-1680 kJ) per day has been promoted for health benefits. ${ }^{9}$ If previously sedentary adults exercised at the lower end of the range (150 kcal [630 kJ] per day) on most (6) days of the week, they would approach the initial health-related goal of $1000 \mathrm{kcal}(4200 \mathrm{~kJ})$ per week. As the duration and intensity of training increase, the number of weekly activity sessions will decrease.

- Example: If a person weighing $70 \mathrm{~kg}$ walked and carried his or her golf clubs while playing golf (4.5 METs), he or she would have to spend about 29 minutes to achieve the minimum health-related goal of $150 \mathrm{kcal}(630 \mathrm{~kJ})$ per day. Mathematically, this is calculated as follows:

- $4.5 \mathrm{METs}=4.5 \mathrm{kcal}$ per $\mathrm{kg}$ per $\mathrm{h}$

Energy expenditure $(\mathrm{kcal} / \mathrm{min})=(4.5 \mathrm{kcal} \times 70 \mathrm{~kg}) \div 60 \mathrm{~min}=5.25 \mathrm{kcal} / \mathrm{min}$

Time $(\mathrm{min} / \mathrm{d})=150 \mathrm{kcal} / \mathrm{d} \div 5.25 \mathrm{kcal}$ per $\min =29 \mathrm{~min} / \mathrm{d}$

- If the same person were interested in accumulating all of his or her weekly exercise through golfing, he or she would need to play about 190 minutes of golf each week. Mathematically, this is calculated as follows:

- Total time $(\mathrm{min} / \mathrm{wk})=1000 \mathrm{kcal} / \mathrm{wk} \div 5.25 \mathrm{kcal} / \mathrm{min}=190 \mathrm{~min} / \mathrm{wk}$

- As participants become more accustomed to regular activity, they should be encouraged to expend 300-400 kcal (1260-1680 kJ) per day ${ }^{9}$ for 3-5 days per week (total energy expenditure 1000-2000 kcal [4200-8400 kJ] per week for adults). 
cise prescriptions based on these tables should be adjusted according to individual responses and other objective measures of intensity (e.g., heart rate and patient's rating of perceived exertion [RPE]).

Some of the limitations associated with the use of standardized tables include differences between people in their baseline levels of fitness, skill, coordination and exercise economy (efficiency), the effects of various environmental factors (e.g., cold, wind, heat, altitude) and differences in the intensity of effort during a particular activity. ${ }^{9}$ As discussed by Shephard, ${ }^{25}$ the standardized tables outlining the metabolic costs associated with various activities are lim-

Table 2: Estimated energy expenditures for common activities as a function of time (data are based on a person who weighs $70 \mathrm{~kg}$ )

Time, min; total energy expenditure, kcal

\begin{tabular}{|c|c|c|c|c|c|c|c|c|c|c|c|c|c|}
\hline \multirow[b]{2}{*}{ Activity } & \multirow[b]{2}{*}{ METs } & \multirow[b]{2}{*}{$\mathrm{EE}$} & & & & & & & & & & & \\
\hline & & & 10 & 15 & 20 & 25 & 30 & 35 & 40 & 45 & 50 & 55 & 60 \\
\hline \multicolumn{14}{|l|}{ Leisure } \\
\hline Backpacking & 7.0 & 0.12 & 82 & 123 & 163 & 204 & 245 & 286 & 327 & 368 & 408 & 449 & 490 \\
\hline Basketball, game & 8.0 & 0.13 & 93 & 140 & 187 & 233 & 280 & 327 & 373 & 420 & 467 & 513 & 560 \\
\hline Basketball, shooting baskets & 4.5 & 0.08 & 53 & 79 & 105 & 131 & 158 & 184 & 210 & 236 & 263 & 289 & 315 \\
\hline Bicycling, general stationary & 7.0 & 0.12 & 82 & 123 & 163 & 204 & 245 & 286 & 327 & 368 & 408 & 449 & 490 \\
\hline $\begin{array}{l}\text { Bicycling, light (10.0-11.9 mph } \\
\text { [16-19.2 kph]) }\end{array}$ & 6.0 & 0.10 & 70 & 105 & 140 & 175 & 210 & 245 & 280 & 315 & 350 & 385 & 420 \\
\hline $\begin{array}{l}\text { Bicycling, moderate }(12.0-13.9 \mathrm{mph} \\
[19.3-22.4 \mathrm{kph}])\end{array}$ & 8.0 & 0.13 & 93 & 140 & 187 & 233 & 280 & 327 & 373 & 420 & 467 & 513 & 560 \\
\hline $\begin{array}{l}\text { Bicycling, vigorous }(14.0-15.9 \mathrm{mph} \\
[22.5-25.6 \mathrm{kph}])\end{array}$ & 10.0 & 0.17 & 117 & 175 & 233 & 292 & 350 & 408 & 467 & 525 & 583 & 642 & 700 \\
\hline Bowling & 3.0 & 0.05 & 35 & 53 & 70 & 88 & 105 & 123 & 140 & 158 & 175 & 193 & 210 \\
\hline $\begin{array}{l}\text { Conditioning exercise, } \\
\text { calisthenics(light to moderate) }\end{array}$ & 3.5 & 0.06 & 41 & 61 & 82 & 102 & 123 & 143 & 163 & 184 & 204 & 225 & 245 \\
\hline $\begin{array}{l}\text { Conditioning exercise, calisthenics } \\
\text { (vigorous) }\end{array}$ & 8.0 & 0.13 & 93 & 140 & 187 & 233 & 280 & 327 & 373 & 420 & 467 & 513 & 560 \\
\hline $\begin{array}{l}\text { Conditioning exercise, stair-treadmill } \\
\text { ergometer }\end{array}$ & 9.0 & 0.15 & 105 & 158 & 210 & 263 & 315 & 368 & 420 & 473 & 525 & 578 & 630 \\
\hline Curling & 4.0 & 0.07 & 47 & 70 & 93 & 117 & 140 & 163 & 187 & 210 & 233 & 257 & 280 \\
\hline Dancing, general aerobic & 6.5 & 0.11 & 76 & 114 & 152 & 190 & 228 & 265 & 303 & 341 & 379 & 417 & 455 \\
\hline Dancing, social or ballroom (fast) & 4.5 & 0.08 & 53 & 79 & 105 & 131 & 158 & 184 & 210 & 236 & 263 & 289 & 315 \\
\hline Fishing, from a boat (sitting) & 2.5 & 0.04 & 29 & 44 & 58 & 73 & 88 & 102 & 117 & 131 & 146 & 160 & 175 \\
\hline Fishing, in a stream (waders) & 6.0 & 0.10 & 70 & 105 & 140 & 175 & 210 & 245 & 280 & 315 & 350 & 385 & 420 \\
\hline Frisbee playing & 3.0 & 0.05 & 35 & 53 & 70 & 88 & 105 & 123 & 140 & 158 & 175 & 193 & 210 \\
\hline Golfing, using a powercart & 3.5 & 0.06 & 41 & 61 & 82 & 102 & 123 & 143 & 163 & 184 & 204 & 225 & 245 \\
\hline Golfing, walking and carrying clubs & 4.5 & 0.08 & 53 & 79 & 105 & 131 & 158 & 184 & 210 & 236 & 263 & 289 & 315 \\
\hline Golfing, walking and pulling clubs & 4.3 & 0.07 & 50 & 75 & 100 & 125 & 151 & 176 & 201 & 226 & 251 & 276 & 301 \\
\hline Hiking, cross-country & 6.0 & 0.10 & 70 & 105 & 140 & 175 & 210 & 245 & 280 & 315 & 350 & 385 & 420 \\
\hline Ice hockey & 8.0 & 0.13 & 93 & 140 & 187 & 233 & 280 & 327 & 373 & 420 & 467 & 513 & 560 \\
\hline Jogging, general & 7.0 & 0.12 & 82 & 123 & 163 & 204 & 245 & 286 & 327 & 368 & 408 & 449 & 490 \\
\hline Playing catch, football or baseball & 2.5 & 0.04 & 29 & 44 & 58 & 73 & 88 & 102 & 117 & 131 & 146 & 160 & 175 \\
\hline Rollerblading, in-line skating & 12.5 & 0.21 & 146 & 219 & 292 & 365 & 438 & 510 & 583 & 656 & 729 & 802 & 875 \\
\hline $\begin{array}{l}\text { Running, } 5.0 \mathrm{mph}(8 \mathrm{kph}) \\
12 \mathrm{~min} / \mathrm{mile}(7.5 \mathrm{~min} / \mathrm{km})\end{array}$ & 8.0 & 0.13 & 93 & 140 & 187 & 233 & 280 & 327 & 373 & 420 & 467 & 513 & 560 \\
\hline $\begin{array}{l}\text { Running, } 7.5 \mathrm{mph}(12 \mathrm{kph}) \text {; } \\
8 \mathrm{~min} / \mathrm{mile}(5 \mathrm{~min} / \mathrm{km})\end{array}$ & 12.5 & 0.21 & 146 & 219 & 292 & 365 & 438 & 510 & 583 & 656 & 729 & 802 & 875 \\
\hline $\begin{array}{l}\text { Running, } 10.9 \mathrm{mph}(17.5 \mathrm{kph}) \text {; } \\
5.5 \mathrm{~min} / \mathrm{mile}(3.4 \mathrm{~min} / \mathrm{km})\end{array}$ & 18.0 & 0.30 & 210 & 315 & 420 & 252 & 630 & 735 & 840 & 945 & 1050 & 1155 & 1260 \\
\hline $\begin{array}{l}\text { Skiing, cross-country, } 4.0-4.9 \mathrm{mph} \\
\text { (6.4-7.9 kph) (moderate) }\end{array}$ & 8.0 & 0.13 & 93 & 140 & 187 & 233 & 280 & 327 & 373 & 420 & 467 & 513 & 560 \\
\hline Skiing, water & 6.0 & 0.10 & 70 & 105 & 140 & 175 & 210 & 245 & 280 & 315 & 350 & 385 & 420 \\
\hline Skiing, downhill & 6.0 & 0.10 & 70 & 105 & 140 & 175 & 210 & 245 & 280 & 315 & 350 & 385 & 420 \\
\hline Snowmobiling & 3.5 & 0.06 & 41 & 61 & 82 & 102 & 123 & 143 & 163 & 184 & 204 & 225 & 245 \\
\hline
\end{tabular}


ited, especially in middle-aged and older people. For instance, exercising at a fixed level of 4 METs (consuming about I4 $\mathrm{mL}$ of oxygen per kilogram per minute) would be an easy level of exercise for a relatively fit person, but it may be near the maximum level for a person with heart failure. Furthermore, with improvements in physical fitness as a result of training, the MET levels would need to be increased to maintain a sufficient training stimulus for further adaptation. ${ }^{20}$ These limitations have led to the use of relative intensity in exercise prescription.

Intensity levels of physical activity in the research laboratory are often expressed relative to oxygen consumption

Table 2 continued

\begin{tabular}{|c|c|c|c|c|c|c|c|c|c|c|c|c|c|}
\hline \multirow[b]{2}{*}{ Activity } & \multirow[b]{2}{*}{ METs } & \multirow[b]{2}{*}{$\mathrm{EE}$} & \multicolumn{11}{|c|}{ Time, min; total energy expenditure, kcal } \\
\hline & & & 10 & 15 & 20 & 25 & 30 & 35 & 40 & 45 & 50 & 55 & 60 \\
\hline Snowshoeing & 8.0 & 0.13 & 93 & 140 & 187 & 233 & 280 & 327 & 373 & 420 & 467 & 513 & 560 \\
\hline Softball, general & 5.0 & 0.08 & 58 & 88 & 117 & 146 & 175 & 204 & 233 & 263 & 292 & 321 & 350 \\
\hline Swimming, general leisure & 6.0 & 0.10 & 70 & 105 & 140 & 175 & 210 & 245 & 280 & 315 & 350 & 385 & 420 \\
\hline Swimming laps, freestyle (vigorous) & 10.0 & 0.17 & 117 & 175 & 233 & 292 & 350 & 408 & 467 & 525 & 583 & 642 & 700 \\
\hline Tennis, general & 7.0 & 0.12 & 82 & 123 & 163 & 204 & 245 & 286 & 327 & 368 & 408 & 449 & 490 \\
\hline Walking, $2.0 \mathrm{mph}$ (3.2 kph) & 2.5 & 0.04 & 29 & 44 & 58 & 73 & 88 & 102 & 117 & 131 & 146 & 160 & 175 \\
\hline Walking, $3.5 \mathrm{mph}$ (5.6 kph) & 3.8 & 0.06 & 44 & 67 & 89 & 111 & 133 & 155 & 177 & 200 & 222 & 244 & 266 \\
\hline Walking, $5.0 \mathrm{mph}(8.0 \mathrm{kph})$ & 8.0 & 0.13 & 93 & 140 & 187 & 233 & 280 & 327 & 373 & 420 & 467 & 513 & 560 \\
\hline \multicolumn{14}{|l|}{ Daily living } \\
\hline Carrying small children & 3.0 & 0.05 & 35 & 53 & 70 & 88 & 105 & 123 & 140 & 158 & 175 & 193 & 210 \\
\hline Chopping wood & 6.0 & 0.10 & 70 & 105 & 140 & 175 & 210 & 245 & 280 & 315 & 350 & 385 & 420 \\
\hline Cleaning house, general & 3.0 & 0.05 & 35 & 53 & 70 & 88 & 105 & 123 & 140 & 158 & 175 & 193 & 210 \\
\hline $\begin{array}{l}\text { Groceries, carrying without shopping } \\
\text { cart }\end{array}$ & 2.5 & 0.04 & 29 & 44 & 58 & 73 & 88 & 102 & 117 & 131 & 146 & 160 & 175 \\
\hline Groceries, carrying upstairs & 7.5 & 0.13 & 88 & 131 & 175 & 219 & 263 & 306 & 350 & 394 & 438 & 481 & 525 \\
\hline Ironing & 2.3 & 0.04 & 27 & 40 & 54 & 67 & 81 & 94 & 107 & 121 & 134 & 148 & 161 \\
\hline Mopping & 3.5 & 0.06 & 41 & 61 & 82 & 102 & 123 & 143 & 163 & 184 & 204 & 225 & 245 \\
\hline Mowing lawn, general & 5.5 & 0.09 & 64 & 96 & 128 & 160 & 193 & 225 & 257 & 289 & 321 & 353 & 385 \\
\hline Raking lawn & 4.3 & 0.07 & 50 & 75 & 100 & 125 & 151 & 176 & 201 & 226 & 251 & 276 & 301 \\
\hline Shovelling snow, manually & 6.0 & 0.10 & 70 & 105 & 140 & 175 & 210 & 245 & 280 & 315 & 350 & 385 & 420 \\
\hline Sweeping floors or carpet & 3.3 & 0.06 & 39 & 58 & 77 & 96 & 116 & 135 & 154 & 173 & 193 & 212 & 231 \\
\hline Sweeping sidewalk & 4.0 & 0.07 & 47 & 70 & 93 & 117 & 140 & 163 & 187 & 210 & 233 & 257 & 280 \\
\hline Vacuuming & 3.5 & 0.06 & 41 & 61 & 82 & 102 & 123 & 143 & 163 & 184 & 204 & 225 & 245 \\
\hline Walking the dog & 3.0 & 0.05 & 35 & 53 & 70 & 88 & 105 & 123 & 140 & 158 & 175 & 193 & 210 \\
\hline Walking, household & 2.0 & 0.03 & 23 & 35 & 47 & 58 & 70 & 82 & 93 & 105 & 117 & 128 & 140 \\
\hline $\begin{array}{l}\text { Walking, pushing/pulling stroller } \\
\text { withchild }\end{array}$ & 2.5 & 0.04 & 29 & 44 & 58 & 73 & 88 & 102 & 117 & 131 & 146 & 160 & 175 \\
\hline Washing dishes & 2.3 & 0.04 & 27 & 40 & 54 & 67 & 81 & 94 & 107 & 121 & 134 & 148 & 161 \\
\hline Watering house plants & 2.5 & 0.04 & 29 & 44 & 58 & 73 & 88 & 102 & 117 & 131 & 146 & 160 & 175 \\
\hline Watering lawn or garden & 1.5 & 0.03 & 18 & 26 & 35 & 44 & 53 & 61 & 70 & 79 & 88 & 96 & 105 \\
\hline Weeding garden & 4.5 & 0.08 & 53 & 79 & 105 & 131 & 158 & 184 & 210 & 236 & 263 & 289 & 315 \\
\hline
\end{tabular}

Note: $M E T$ = metabolic equivalent $(1 \mathrm{MET}=3.5 \mathrm{~mL}$ oxygen per kilogram per minute, or $1 \mathrm{kcal}$ [4.2 $\mathrm{kJ}$ ] per kilogram per hour), EE = energy expenditure (kilocalories) per kilogram per minute.

*Data were derived from the Compendium of Physical Activities. ${ }^{23,24}$ The latest version of this compendium ${ }^{23}$ contains absolute energy expenditures associated with 605 different physical activities, including activities of daily living.

Guidelines to using this chart

- The minimum weekly energy expenditure generally advocated is $1000 \mathrm{kcal}(4200 \mathrm{~kJ}) .9,21$ The recommended daily energy expenditure is $150-400 \mathrm{kcal}(630-1680 \mathrm{~kJ}) .{ }^{9}$ If previously sedentary adults exercised at the lower end of the recommendation ( $150 \mathrm{kcal}$ [ $630 \mathrm{~kJ}$ ] per day) on most (6) days of the week, they would approach the initial health-related goal of $1000 \mathrm{kcal}(4200 \mathrm{~kJ})$ per week.

- Example: If a person weighing $70 \mathrm{~kg}$ walked and carried his or her golf clubs while playing golf ( $4.5 \mathrm{METs}$ ), he or she would expend $158 \mathrm{kcal}$ ( $664 \mathrm{~kJ})$ for every 30 minutes of activity. Mathematically, this is calculated as follows:

- Energy expenditure $(\mathrm{kcal})=4.5 \mathrm{METs} \times 70 \mathrm{~kg} \times(30 \mathrm{~min} \sqrt{ } 60 \mathrm{~min})=158 \mathrm{kcal}$

- If the same person were interested in accumulating all of his or her weekly exercise through golfing, he or she would need to play 190 minutes of golf each week.

Energy expenditure $(\mathrm{kcal} / \mathrm{min})=158 \mathrm{kcal}$ per session $\sqrt{ } 30 \mathrm{~min}=5.25 \mathrm{kcal} / \mathrm{min}$

- Total time $(\mathrm{min} / \mathrm{wk})=1000 \mathrm{kcal} / \mathrm{wk} \sqrt{ } 5.25 \mathrm{kcal} / \mathrm{min}=190 \mathrm{~min} / \mathrm{wk}$ 
Box 3: Examples of individualized exercise prescriptions for increasing physical activity levels

Patient A: A 70-kg woman aged 68 years with no previous exercise pattern. She has no physical limitations to exercise Goal: To start an exercise program and try to maintain it Preferred activities: Low-intensity exercise (walking and gardening), 7 days per week

Target: 1000 kcal (4200 kJ) per week

Exercise prescription:

- Walking for $30 \mathrm{~min}$ daily at a speed of $2 \mathrm{mph}(3.2 \mathrm{kph})$ $=88 \mathrm{kcal}(370 \mathrm{~kJ})$ per session

- Weeding the garden for 30 min twice weekly = $158 \mathrm{kcal}(664 \mathrm{~kJ})$ per session

- Watering the garden for 20 min twice weekly $=44 \mathrm{kcal}(185 \mathrm{~kJ})$ per session

Patient B: A 70-kg man aged 52 years who had been active in university. He has no physical limitations to exercise and travels frequently

Goal: To rekindle interest in exercise and try to maintain it Preferred activities: Moderate-intensity exercise (walking, swimming and playing tennis), 3-5 days per week

Target: $1000 \mathrm{kcal}(4200 \mathrm{~kJ})$ per week

Exercise prescription:

- Walking for 30 min 5 days per week at a speed of $3.5 \mathrm{mph}$ $(5.6 \mathrm{kph})=133 \mathrm{kcal}(559 \mathrm{~kJ})$ per session

- Swimming for $30 \mathrm{~min}$ twice weekly $=210 \mathrm{kcal}(882 \mathrm{~kJ})$ per session

- Playing tennis for 60 min once weekly $=490 \mathrm{kcal}(2058 \mathrm{~kJ})$

$\left(\mathrm{VO}_{2}\right)$. However, the assessment of $\mathrm{VO}_{2}$ is usually not feasible or practical outside of the laboratory. Heart rate is a practical, objective measure of exercise work rates. Ideally, before starting an exercise program, a person should have his or her maximum heart rate determined during an incremental to maximal exercise test. If this is not feasible, then the maximum heart rate can be estimated based on the standardized equations in Box 4. There are some concerns, however, regarding the accuracy of these equations for obese people ${ }^{11}$ and women. In fact, in our practice we have found that, for women, 226 - age (in years) provides a better estimate of the maximum heart rate than 220 - age and therefore is more appropriate for exercise prescriptions (unpublished observations). Others have also created equations for obese people. ${ }^{11}$

Determination of a person's maximum heart rate provides an easy means of estimating the training heart rate range in most cases (Box 5). As such, prescribing exercise according to a percentage of a person's maximum heart rate has been, and remains, the primary technique used by health and fitness professionals. However, it is preferable to establish levels of exercise intensity on the basis of a person's heart rate reserve rather than merely a percentage of his or her maximum heart rate (Box 6). The heart rate reserve takes into account both the maximum and the resting heart rates. The training heart rate range can differ markedly depending on whether it is calculated using the percentage of the maximum
Box 4: Equations for predicting a person's maximum heart rate

Men: 220 - age

Women: 226 - age

Obese people: $220-(0.5 \times$ age $)$

heart rate (as in Box 5) or the percentage of the heart rate reserve (as in Box 6). These differences are especially apparent with different resting heart rates. Therefore, because the resting heart rate varies a great deal from person to person, it is better to prescribe levels of exercise intensity according to a person's heart rate reserve.

Subjective indicators of the relative intensity of effort are shown in Table 3. For instance, participants are often prescribed exercise that they perceive to be of moderate intensity. The most commonly used scale is the RPE (rating of perceived exertion) scale. ${ }^{27,28}$ Initially, the 15 -category scale was used (Table 4); however, the Io-point category-ratio RPE is being used increasingly, especially in cardiac rehabilitation settings. Although subjective, the RPE scale does have advantages, especially for patients using medications that affect heart rate (as discussed later). The use of the RPE scale is also an important means of prescribing exercise to people when no equipment is available for physiologic assessment (e.g., heart rate monitors).

Health and fitness agencies have also started to design exercise programs according to a person's feelings regarding his or her breathing and body temperature. Although limited in their ability to precisely define training ranges, these subjective scales are easy to understand and use, which makes them useful for the general population (Table 3 ).

An example of a 7-month exercise prescription that incorporates objective and subjective indicators of exercise intensity is provided in Table 5 for a healthy adult starting an exercise program. We currently use this template for prescribing exercise to patients. It is a variation of the model proposed by the American College of Sports Medicine. ${ }^{9}$

The guidelines discussed above for prescribing levels of exercise intensity are generally appropriate for young to middle-aged adults. As with fitness testing, special considerations should be taken when prescribing exercise for other groups, such as children, elderly people, overweight or obese patients, and patients with chronic disease. For instance, the general exercise prescriptions for adults appear to be appropriate in most circumstances for children over the age of 6 years..$^{15,29}$ The American College of Sports Medicine does not recommend prescribing an upper heart rate limit for children because they are at very low risk of cardiac events and are able to adjust exercise according to their tolerance levels. ${ }^{29}$ Children will often prefer play activities over formal training programs and frequently engage in sporadic rather than continuous activities. It is recommended that children engage in activities that stress the major muscle groups and tax both the cardiovascular and musculoskeletal systems. 
For elderly people, although the exercise prescription for healthy adults is generally applicable, there are several special considerations that should be taken. The maintenance of independent living is of primary concern in elderly populations. In fact, many elderly people may be at or near the functional threshold for dependence. Elderly people often display markedly reduced aerobic and musculoskeletal fitness, with the latter being particularly important in determining functional status..$^{5,6}$ With a further worsening of musculoskeletal fitness, a person may lose the ability to live an independent lifestyle. Several researchers, including our own research group, have actively promoted the need for elderly people to improve their musculoskeletal fitness. Depending on the frailty of the patient, it is often advisable to start a training program with flexibility exercises, building up to resistance exercises and then aerobic exercises..$^{30}$ Resistance and flexibility training should be encouraged for at least 2 days per week. Walking exercise in a group setting is often a preferable aerobic activity for elderly people. ${ }^{9}$ The use of repeat short (Io-minute) bouts of exercise throughout the day is often desirable. The recommendation of 30 minutes of moderate-intensity exercise on most days of the week is appropriate for elderly people.

The benefits of exercise for the treatment of obesity are clear. There have been several recommendations regarding the optimal exercise prescription for overweight and obese people. ${ }^{31}$ In general, overweight people should engage in at least 30 minutes of moderate-intensity physical activity on most (preferably all) days of the week. ${ }^{20}$ They are also advised to expend about 250-300 kcal (I050-I260 kJ) per session. ${ }^{15}$ As recently reviewed by Jakicic, ${ }^{32}$ moderate-intensity exercise lasting 45 to 60 minutes per day is likely required for weight control or reduction. A good practical prescription for over-

Box 5: Examples of training ranges calculated on the basis of intensity level of exercise and maximum heart rate $\left(\mathrm{HR}_{\max }\right)$

Light-intensity exercise $\left(45 \%-54 \% \mathrm{HR}_{\max }\right)$

Example: 60-yr-old woman

- $\mathrm{HR}_{\max }(226-$ age $)=226-60=166$ beats $/ \mathrm{min}$

- $45 \%$ of $\mathrm{HR}_{\max }=75$ beats $/ \mathrm{min}$

- $54 \%$ of $\mathrm{HR}_{\max }=90$ beats $/ \mathrm{min}$

- Training range $=75-90$ beats $/ \mathrm{min}$

Moderate-intensity exercise $\left(55 \%-69 \% \mathrm{HR}_{\max }\right)$

Example: 45-yr-old man

- $\mathrm{HR}_{\max }(220-$ age $)=220-45=175$ beats $/ \mathrm{min}$

- $55 \%$ of $\mathrm{HR}_{\max }=96$ beats $/ \mathrm{min}$

- $69 \%$ of $\mathrm{HR}_{\max }=121$ beats $/ \mathrm{min}$

- Training range $=96-121$ beats $/ \mathrm{min}$

High-intensity exercise $\left(70 \%-89 \% \mathrm{HR}_{\max }\right)$

Example: 63-yr-old man

- $\mathrm{HR}_{\max }(220-$ age $)=220-63=157$ beats $/ \mathrm{min}$

- $70 \%$ of $\mathrm{HR}_{\max }=110$ beats $/ \mathrm{min}$

- $89 \%$ of $\mathrm{HR}_{\max }=140$ beats $/ \mathrm{min}$

- Training range $=110-140$ beats $/ \mathrm{min}$ weight and obese adults is to have them start slowly (e.g., walking for to minutes) and to build up to 30 minutes per day for 5 days per week. Increasing the duration of activity to 60 minutes per day may be considered when the patient has built up a tolerance to 30 minute per day. ${ }^{32}$

People with chronic disease should have specific programs developed by a health care professional (e.g., a physician or exercise therapist, or both) that are tailored to and appropriate for their disease status. Special attention must be paid to the severity of the patient's condition. Physicians should regard an exercise prescription the same as prescribing a medication: What is the optimal dose for this patient?

The general principles for healthy adults can be applied in the training of patients with cardiac disease. For instance, the use of the heart rate reserve is advisable for many such patients. Also, patients with cardiac disease should engage in 20-6o minutes of exercise on most days of the week. An energy expenditure of about $\mathrm{r} 600 \mathrm{kcal}(6720 \mathrm{~kJ})$ per week has been shown to effectively halt the progression of coronary artery disease, and an expenditure of $2200 \mathrm{kcal}(9240 \mathrm{~kJ})$ per week has been associated with plaque reduction and a reversal of the disease. ${ }^{33,34}$

There are, however, slight differences in the exercise prescription model for patients with cardiac disease. For instance, it is generally advocated that such patients be moni-

Box 6: Examples of training ranges calculated on the basis of intensity level of exercise and the heart rate reserve (HRR)

Light-intensity exercise (30\%-39\% of HRR)

Example: 60-yr-old woman

- $\mathrm{HR}_{\max }(226-$ age $)=226-60=166$ beats $/ \mathrm{min}$

- Resting $H R\left(\mathrm{HR}_{\text {rest }}\right)=90$ beats $/ \mathrm{min}$

- $\mathrm{HRR}=\left[\left(\mathrm{HR}_{\max }-\mathrm{HR}_{\text {rest }}\right) \times 30 \%\right.$ or $\left.39 \%\right]+\mathrm{HR}_{\text {rest }}$ $30 \%$ of HRR $=(166-90) \times 0.30)+90=113$ beats $/ \mathrm{min}$

$39 \%$ of HRR $=(166-90) \times 0.39)+90=120$ beats $/ \mathrm{min}$

- Training range $=118-127$ beats $/ \mathrm{min}$

Moderate-intensity exercise (40\%-59\% HRR)

Example: 45-yr-old man

- $\mathrm{HR}_{\max }(220-$ age $)=220-45=175$ beats $/ \mathrm{min}$

- $\mathrm{HR}_{\text {rest }}=80$ beats $/ \mathrm{min}$

- $\mathrm{HRR}=\left[\left(\mathrm{HR}_{\max }-\mathrm{HR}_{\text {rest }}\right) \times 40 \%\right.$ or $\left.59 \%\right]+\mathrm{HR}_{\text {rest }}$

$40 \%$ of HRR $=(175-80) \times 0.40)+80=118$ beats $/ \mathrm{min}$

$59 \%$ of HRR $=(175-80) \times 0.59)+80=136$ beats $/ \mathrm{min}$

- Training range $=118-136$ beats $/ \mathrm{min}$

High-intensity exercise (60\%-84\% HRR)

Example: 63-yr-old man

- $\mathrm{HR}_{\max }(220-$ age $)=220-63=157$ beats $/ \mathrm{min}$

- $\mathrm{HR}_{\text {rest }}=84$ beats $/ \mathrm{min}$

- $\mathrm{HRR}=\left[\left(\mathrm{HR}_{\max }-\mathrm{HR}_{\text {rest }}\right) \times 60 \%\right.$ or $\left.84 \%\right]+\mathrm{HR}_{\text {rest }}$

$60 \%$ of HRR $=(157-84) \times 0.60)+84=128$ beats $/ \mathrm{min}$

$84 \%$ of HRR $=(157-84) \times 0.84)+84=145$ beats $/ \mathrm{min}$

- Training range $=130-149$ beats $/ \mathrm{min}$ 
tored for arrhythmias and abnormal blood pressure responses during the initial stages of training. The length of supervision depends on the clinical status of the individual patient and the time it takes to establish safety during the rehabilitation program (often 6-I2 sessions). Also, many patients with cardiac disease are extremely deconditioned and cannot tolerate extended periods of continuous exercise, especially early into a program. Patients with lower functional status require a more conservative exercise intervention. ${ }^{9}$ For these patients, it is advocated that the recommended volume of physical activity be achieved through multiple exercise sessions per day. The duration of each session depends on the clinical status of the patient. ${ }^{35}$ In our practice, it is not uncommon for lower-function patients (e.g., those with heart failure) to engage in 3-4 sessions of low-intensity aerobic exercise per day, each session lasting $3-5$ minutes. The duration of each session can be increased progressively, depending on the individual patient, until the recommended volume of exercise is achieved. ${ }^{35}$

The minimal training intensity threshold is about $45 \%$ of the heart rate reserve for patients with coronary artery disease, ${ }^{34}$ compared with $30 \%$ of the heart rate reserve for unfit healthy people. ${ }^{18}$ This difference is thought to be the result of the difficulty for cardiac patients in achieving true maximum effort during a stress test. ${ }^{18} \mathrm{~A}$ similar intensity is used for patients with heart failure when they begin many traditional rehabilitation programs. Additional benefits, however, are likely achieved with higher exercise intensities, if tolerated and safe for the patient. ${ }^{34}$ In fact, we prescribe exercise training intensities of about $65 \%$ of the heart rate reserve for many patients with cardiac disease. ${ }^{7}$ Interval training has also been shown to be effective for patients with coronary artery disease $^{7}$ and heart failure. ${ }^{36}$

A physician's ability to prescribe exercise intensity based on the patient's RPE (rating of perceived exertion) or dyspnea is important. Often patients have blunted chronotropic responsiveness to exercise (e.g., heart failure, transplant) or are taking medications that affect heart rate. In these patients, the physician can comfortably use the RPE to prescribe exercise.

\section{Other techniques for evaluating physical activity levels and intensity}

\section{Cardiac monitors}

Many health and fitness professionals rely on heart rate monitors to objectively monitor exercise intensity and establish training workloads for individual patients. Heart rate monitors cost from $\$ 50$ to $\$ 500$, are lightweight and are user friendly, qualities that make them appropriate for wide-scale use. During exercise, there is a relatively linear relation between heart rate, $\mathrm{VO}_{2}$ and energy expenditure.

Table 4: Category scale for rating perceived exertion (RPE) and category-ratio RPE scale

\begin{tabular}{|c|c|c|c|}
\hline \multicolumn{2}{|c|}{ Category RPE scale } & \multicolumn{2}{|c|}{ Category-ratio RPE scale } \\
\hline Score & Level of exertion & Score & Level of exertion \\
\hline 6 & None & 0 & None \\
\hline 7 & Extremely light & 0.5 & $\begin{array}{l}\text { Very, very weak } \\
\text { (just noticeable) }\end{array}$ \\
\hline \multicolumn{4}{|l|}{8} \\
\hline 9 & Very light & 1 & Very weak \\
\hline 10 & & 2 & Weak (light) \\
\hline 11 & Light & 3 & Moderate \\
\hline 12 & & 4 & Somewhat strong \\
\hline 13 & Somewhat hard & 5 & Strong (heavy) \\
\hline 14 & & 6 & \\
\hline 15 & Hard (heavy) & 7 & Very strong \\
\hline 16 & & 8 & \\
\hline 17 & Very hard & 9 & \\
\hline 18 & & 10 & $\begin{array}{l}\text { Very, very strong } \\
\text { (almost maximum) }\end{array}$ \\
\hline 19 & Extremely hard & & Maximum \\
\hline 20 & Maximum exertion & & \\
\hline
\end{tabular}

Sources: Borg ${ }^{27}$ and Noble et al..$^{28}$

Table 3: Relative intensities for aerobic exercise prescription (for activities lasting up to 60 minutes)*

\begin{tabular}{|c|c|c|c|c|c|c|c|c|}
\hline Intensity & & $\begin{array}{c}\% \\
\text { HRR }\end{array}$ & $\begin{array}{c}\% \\
H R_{\max }\end{array}$ & $\begin{array}{l}\text { 15-category } \\
\text { RPE scale† }\end{array}$ & $\begin{array}{l}\text { Category- } \\
\text { ratio RPE } \\
\text { scale† }\end{array}$ & Breathing rate & $\begin{array}{c}\text { Body } \\
\text { temperature }\end{array}$ & $\begin{array}{l}\text { Example } \\
\text { of activity }\end{array}$ \\
\hline Very light effort & & $<20$ & $<35$ & $<10$ & $<2$ & Normal & Normal & Dusting \\
\hline Light effort & \multirow{3}{*}{$\begin{array}{l}\text { Range } \\
\text { required } \\
\text { for } \\
\text { health }\end{array}$} & $20-39$ & $35-54$ & $10-11$ & $2-3$ & Slight increase & Start to feel warm & Light gardening \\
\hline Moderate effort & & $40-59$ & $55-69$ & $12-13$ & $4-6$ & Greater increase & Warm & Brisk walking \\
\hline Vigorous effort & & $60-84$ & $70-89$ & $14-16$ & $7-8$ & More out of breath & Quite warm & Jogging \\
\hline Very hard effort & & $>84$ & $>89$ & $17-19$ & 9 & Greater increase & Hot & Running fast \\
\hline Maximal effort & & 100 & 100 & 20 & 10 & $\begin{array}{l}\text { Completely out of } \\
\text { breath }\end{array}$ & $\begin{array}{l}\text { Very hot, } \\
\text { perspiring heavily }\end{array}$ & Sprinting all-out \\
\hline
\end{tabular}

Note: $\mathrm{HRR}=$ heart rate reserve, $\mathrm{HR}_{\max }=$ maximum heart rate, $\mathrm{RPE}=$ patient's rating of perceived exertion.

${ }^{*}$ Created from information provided in the handbook for Canada's Physical Activity Guide to Healthy Active Living, ${ }^{26}$ and the American College of Sports Medicine's guidelines for exercise testing and prescription. ${ }^{9}$

†See Table 4 for details about the RPE scales. 
Therefore, many have argued that heart rate monitors are also useful in determining physical activity levels and patterns during "free-living" activities. As such, several investigators, including our research group, use heart rate monitors to assess activity patterns objectively over prolonged periods. There are a variety of ways to evaluate physical activity from heart rate data. ${ }^{37-39}$

The use of heart rate monitors appears to be particularly helpful for monitoring physical activity of moderate intensity throughout the day and activities of high intensity when they occur. However, the relation between heart rate and $\mathrm{VO}_{2}$ is not strong during low-intensity or sedentary activities $^{38}$ and high-intensity exercise. ${ }^{37}$ In addition, there are several sources of error in the measurement of physical activity through heart rate monitoring. ${ }^{37-39}$ For instance, heart rate is influenced by high ambient temperature, emotional stress, high humidity, caffeine, medications, dehydration, postural position, the size of the muscle mass involved in exercise, fatigue and illness. ${ }^{37-39}$ Despite these limitations, heart rate monitoring has been shown to be a valid and reliable means of determining physical activity, especially patterns of activity. However, it is clearly not the optimal means of assessing daily physical activity. Heart rate monitors are now often used in conjunction with other measures of physical activity (e.g., questionnaires and pedometers) to compensate for some of the inherent limitations with this technology.

\section{Motion sensors}

Motion sensors are effective for monitoring body movement and as such provide an objective estimate of physical activity.
Motion sensors range in type from simple pedometers (discussed in the next section) to more sophisticated triaxial accelerometers. Motion detectors have been evaluated extensively in recent years regarding their accuracy and reliability for the assessment of physical activity. Several good reviews on the topic are available. ${ }^{37-39}$ Accelerometers involve the measurement of acceleration of the limbs and trunk, with uniaxial devices measuring in 1 plane and triaxial devices in 3 planes. ${ }^{37}$ Currently, the use of accelerometers is generally confined to research applications. With further advancements in the technology and reductions in cost, this equipment will probably be used increasingly for health promotion.

\section{Pedometers}

Pedometers have been used increasingly in the evaluation of physical activity and in exercise prescription. Recently, activity guidelines (as reviewed by Tudor-Locke and Bassett ${ }^{40}$ ) have been proposed based on the number of steps taken per day (Box 7). For exercise prescription, an increase of 3000-4000 steps per day taken during 30 minutes of moderate-intensity walking (above that of activities of daily living) has been proposed to meet current public health recommendations.$^{40}$ This level of exercise has been shown to lead to health benefits in a community setting. ${ }^{41}$ However, some may find this change in activity (steps per day) hard to achieve early into an exercise program. Therefore, patients may prefer to begin a pedometer-based walking program by adding 500 steps per day for each week of exercise until they reach their desired goal of 3000-4000 additional steps per day.

Table 5: An example of a 7-month exercise program for a healthy adult

\begin{tabular}{|c|c|c|c|c|c|c|c|}
\hline \multirow[b]{2}{*}{ Program stage } & \multirow{2}{*}{$\begin{array}{l}\text { Length of } \\
\text { program, wk }\end{array}$} & \multirow{2}{*}{$\begin{array}{l}\text { Frequency, } \\
\text { days/wk }\end{array}$} & \multicolumn{4}{|c|}{ Intensity } & \multirow{2}{*}{$\begin{array}{l}\text { Time per } \\
\text { session, min }\end{array}$} \\
\hline & & & $\% \mathrm{HR}_{\max }$ & $\% \mathrm{HRR}$ & $\mathrm{RPE}^{*}$ & Breathing rate & \\
\hline Initial stage & 1 & 3 & $55-65$ & $40-50$ & $2-4$ & Slightly increased & $15-20$ \\
\hline \multirow{3}{*}{$\begin{array}{l}\text { Perform light muscular } \\
\text { endurance activities } \\
\text { - Engage in aerobic } \\
\text { exercise of light to } \\
\text { moderate intensity }\end{array}$} & 2 & 3 & $55-65$ & $40-50$ & $2-4$ & Slightly increased & $20-25$ \\
\hline & 3 & 3 & $65-70$ & $50-60$ & $3-5$ & Noticeably increased & $20-25$ \\
\hline & 4 & 3 & $65-70$ & $50-60$ & $3-5$ & Noticeably increased & $25-30$ \\
\hline Improvement & $5-7$ & 4 & $70-75$ & $60-70$ & $3-5$ & Noticeably increased & $25-30$ \\
\hline \multirow{5}{*}{$\begin{array}{l}\text { - Increase exercise } \\
\text { intensity and duration } \\
\text { with improved fitness } \\
\text { - Try to achieve health } \\
\text { and fitness goals }\end{array}$} & $8-10$ & 4 & $70-75$ & $60-70$ & $3-5$ & Noticeably increased & $30-35$ \\
\hline & $11-13$ & $3-5$ & $75-80$ & $65-75$ & $4-6$ & Noticeably increased & $30-35$ \\
\hline & $14-16$ & $3-5$ & $75-80$ & $65-75$ & $4-6$ & Noticeably increased & $30-35$ \\
\hline & $17-20$ & $3-5$ & $75-85$ & $70-80$ & $4-8$ & $\begin{array}{l}\text { More difficulty talking } \\
\text { while exercising }\end{array}$ & $35-40$ \\
\hline & $21-24$ & $3-5$ & $75-85$ & $70-80$ & $4-8$ & $\begin{array}{l}\text { More difficulty talking } \\
\text { while exercising }\end{array}$ & $35-40$ \\
\hline \multicolumn{8}{|l|}{ Maintenance } \\
\hline $\begin{array}{l}\text { - Try to maintain health- } \\
\text { related fitness }\end{array}$ & $24-28$ & $3-5$ & $75-85$ & $70-80$ & $4-8$ & $\begin{array}{l}\text { More difficulty talking } \\
\text { while exercising }\end{array}$ & $30-45$ \\
\hline
\end{tabular}

Note: $\mathrm{HR}_{\max }=$ maximum heart rate, $\mathrm{HRR}=$ heart rate reserve, $\mathrm{RPE}=$ patient's rating of perceived exertion.

${ }^{*}$ The values are based on the 10-point category-ratio RPE scale (see Table 4). 
Box 7: Physical activity levels as they relate to number of steps per day

- Sedentary $<5000$

- Low level of activity (i.e., daily activity) 5 000-7 499

- Somewhat active 7 500-9 999

- Active $10000-12499$

- Highly active $>12500$

Because of the ease of use and low cost $(\$ 15-\$ 30)$ of pedometers, the use of this technology may represent a significant breakthrough in health promotion through physical activity. Common pedometers (e.g., the Yamax Digi-walker) have been shown to have high accuracy and reliability ${ }^{42,43}$ during walking activities. ${ }^{44-46}$ Pedometers are, however, limited at walking paces that are lower $(<60 \mathrm{~m} / \mathrm{min})$ than the normal walking speed of the general population. ${ }^{47}$ Thus, they may not be suitable for use by older people who have slow or shuffling gaits. Furthermore, pedometers do not provide information regarding the intensity or pattern of activities and are not useful during activities such as cycling and weight lifting. ${ }^{38}$ Despite these limitations, the use of pedometers in health promotion will surely increase. The step-count guidelines as outlined above provide an important opportunity for eliciting behavioural change.

\section{New technologies}

It is foreseeable that heart monitors, motion sensors and new technologies (e.g., global positioning systems) will be used increasingly in large population-based studies to further our insight into the type, quantity and quality of exercise required for optimal health benefits. We anticipate that a recent new line of portable global positioning systems will be used progressively more in research and by the general public. For instance, the current Forerunner $30 \mathrm{I}$ by Garmin (which retails for about \$300) allows for the concurrent assessment of distance travelled, speed of movement and heart rate over a prolonged period in multiple-activity environments (e.g., cycling, kayaking, running and walking). The ease of use of these devices and the information provided are attractive features of global positioning systems. However, the cost of the individual units still remains prohibitive.

\section{Fitness machines}

The majority of fitness machines (commonly found in fitness clubs) provide an estimate of energy expenditure and intensity (METs, watts, kilocalories per minute). Accordingly, many people use this type of equipment to estimate their intensity of exercise and energy expenditure. This form of equipment is extensively used in exercise rehabilitation settings. However, more objective measures of intensity (e.g., heart rate monitoring) are usually also used, in large part because of the difficulties in attaining accurate and reli- able workloads on fitness equipment. The work rate and energy expenditures provided by such machines are often rough estimates of the actual values. Furthermore, if the reliability of these machines is low, then their utility for exercise prescription is low. It is therefore recommended that people using fitness equipment also incorporate some other measure (e.g., heart rate) to define their work intensity more objectively.

\section{Available resources}

Health Canada, the Canadian Society for Exercise Physiology, the American College of Sports Medicine, the Heart and Stroke Foundation of Canada and the American Heart Association have all been instrumental in the dissemination of information regarding the health benefits of physical activity. In North America, numerous resources are available for the general population and physicians alike. Summaries of available free resources are included in Box 8.

Box 8: Free resources available to physicians and patients

- Physical activity guides: Health Canada, in collaboration with the Canadian Society for Exercise Physiology, has created a series of physical activity guides and information booklets for people of all ages. These guides and handbooks provide a general synopsis of expert opinion on the importance of physical activity for optimal health status and provide simple to follow recommendations for change. They are available at www.phac-aspc.gc.ca/pau-uap/fitness/downloads.html

- Canadian Society for Exercise Physiology (www.csep.ca): Free resources for people interested in learning more about the health benefits of physical activity and engaging in physical activity. Examples include pre-exercise screening forms (PAR-Q and PAR-medX [www.csep.ca/forms.asp]), information about advanced health and fitness certifications, and guidelines for exercise during pregnancy and the postpartum period (www.csep.ca/pdfs/joint\%20sogc_csep\%20guidelines.pdf)

- Heart and Stroke Foundation of Canada (ww2.heartandstroke.ca): Articles on the health benefits of physical activity

- American Heart Association (www.americanheart.org): Free resources that promote the health benefits of physical activity and provide simple to follow recommendations. Examples include the Just Move Personal Fitness Center (www.justmove.org), which provides registered clients with exercise recommendations, fitness resources and the ability to track their daily activity levels; Top Ten Ways to Help Children Develop Healthy Habits (www.americanheart.org /presenter.jhtml?identifier=3030485); Get Fit and Eat Healthy (www.americanheart.org/presenter.jhtml ?identifier $=3017008$ )

- American College of Sports Medicine (www.ascm.org): Free resources for people interested in health and fitness. Also available are important commentaries on a variety of topics (www.acsm.org/health\%2Bfitness/comments.htm), guidelines for aerobic activity (www.acsm.org/pdf /Guidelines.pdf) and a newsletter designed for the general public (www.acsm.org/health\%2Bfitness/fit_society.htm) 


\section{Summary}

Regular physical activity is important for the primary and secondary prevention of several chronic diseases. Significant health benefits can be attained through exercise of light to moderate intensity on most days of the week. In fact, exercise prescription for health does not need to be complicated and can revolve around many activities of daily living. People should choose exercises and activities that they prefer and should try to do them on most days of the week for about 20-60 minutes. The higher the intensity of activity, the less time required. Structured physical training is not required for health benefits to occur, and physical activity can be accumulated throughout the day, even through short (Io-minute) bouts of exercise. The goal of accumulating about rooo kcal (4200 kJ) of energy expenditure per week through physical activity is a good general health guideline. However, health benefits may also be seen at lower levels of energy expenditure, especially in people who are extremely deconditioned or elderly. Physical activity is appropriate and should be encouraged for people of all ages.

This article has been peer reviewed.

From the School of Human Kinetics, University of British Columbia (Warburton, Nicol, Bredin), and the Healthy Heart Program, St. Paul's Hospital (Warburton, Nicol), Vancouver, BC

Competing interests: None declared.

Contributors: Darren Warburton was responsible for the conception of the review and the writing of the manuscript. Shannon Bredin provided assistance with the writing of the manuscript and background research. Crystal Whitney Nicol provided assistance with background research. All of the authors critically reviewed the article for intellectual content and approved the final version.

Acknowledgements: This research was supported by the Michael Smith Foundation for Health Research, the Canadian Institutes of Health Research, the Canada Foundation for Innovation and the BC Knowledge Development Fund.

\section{REFERENCES}

I. Warburton DER, Nicol CW, Bredin SSD. Health benefits of physical activity: the evidence [review]. CMAJ 2006;174(6):80I-9.

2. Canadian Society for Exercise Physiology. Canadian physical activity, fitness and lifestyle approach. 3 rd ed. Ottawa: The Society; 2003.

3. American College of Sports Medicine. ACSM's health-related physical fitness assessment manual. Baltimore: Lippincott Williams \& Wilkins; 2005.

4. Canadian Society for Exercise Physiology. The Certified Fitness Appraiser Resource Manual. Gloucester (ON): Canadian Society for Exercise Physiology; I993.

5. Warburton DE, Gledhill N, Quinney A. The effects of changes in musculoskeletal fitness on health. Can J AppI Physiol 2001;26:I6I-216.

6. Warburton DE, Gledhill N, Quinney A. Musculoskeletal fitness and health. Can J Appl Physiol 2001;26:217-37.

7. Warburton DE, McKenzie DC, Haykowsky MJ, et al. Effectiveness of high-intensity interval training for the rehabilitation of patients with coronary artery disease. Am JCardiol 2005;95:1080-4.

8. Leger LA, Mercier D, Gadoury C, et al. The multistage 20 metre shuttle run test for aerobic fitness. J Sports Sci ig88;6:93-Ior.

9. American College of Sports Medicine. ACSM's Guidelines for exercise testing and prescription. 6th ed. Philadelphia: Lippincott Williams \& Wilkins; 2000.

Io. American College of Sports Medicine. ACSM's Guidelines for exercise testing and prescription. 6th ed. Philadelphia: Lippincott Williams \& Wilkins; 2000. p. 225.

II. Miller WC, Wallace JP, Eggert KE. Predicting max $\mathrm{HR}$ and the $\mathrm{HR}^{-\mathrm{VO}_{2}}$ relationship for exercise prescription in obesity. Med Sci Sports Exerc 1993;25:1077-8I.

I2. Wannamethee SG, Shaper AG, Walker M. Physical activity and mortality in older men with diagnosed coronary heart disease. Circulation 2000;102:1358-63.

I3. Wannamethee SG, Shaper AG, Walker M. Changes in physical activity, mortality, and incidence of coronary heart disease in older men. Lancet 1998;351:1603-8.

I4. National Population Health Survey. 1998/gg. Ottawa: Statistics Canada; 2001.
15. American College of Sports Medicine. Position stand: The recommended quantity and quality of exercise for developing and maintaining cardiorespiratory and muscular fitness, and flexibility in healthy adults. Med Sci Sports Exerc 1998;30:975-91.

I6. Shephard RJ. Absolute versus relative intensity of physical activity in a dose-response context. [discussion S4I9-20]. Med Sci Sports Exerc 200I;33:S400-18.

I7. Warburton DE, Sheel AW, Hodges AN, et al. Effects of upper extremity exercise training on peak aerobic and anaerobic fitness in patients after transplantation. Am J Cardiol 2004;93:939-43.

18. Swain DP, Franklin BA. VO(2) reserve and the minimal intensity for improving cardiorespiratory fitness. Med Sci Sports Exerc 2002;34:152-7.

I9. Physical activity and health: a report of the Surgeon General. Atlanta: US Department of Health and Human Services, US Centers for Disease Control and Prevention, National Center for Chronic Disease Prevention and Health Promotion; 1996.

20. Nieman DC. Exercise testing and prescription: a health-related approach. $4^{\text {th }} \mathrm{ed}$ London: Mayfield Publishing Company; I999.

2I. Lee IM, Skerrett PJ. Physical activity and all-cause mortality: what is the dose-response relation? [discussion S493-4]. Med Sci Sports Exerc 2001;33:S459-7I.

22. Myers J, Kaykha A, George S, et al. Fitness versus physical activity patterns in predicting mortality in men. Am JMed 2004;117:912-8.

23. Ainsworth BE, Haskell WL, Whitt MC, et al. Compendium of physical activities: an update of activity codes and MET intensities. Med Sci Sports Exerc 2000;32: S498-504.

24. Ainsworth BE, Haskell WL, Leon AS, et al. Compendium of physical activities: classification of energy costs of human physical activities. Med Sci Sports Exerc I993;25:7I-80.

25. Shephard RJ. Limits to the measurement of habitual physical activity by questionnaires. [discussion 206]. Br J Sports Med 2003;37:197-206.

26. Canada's physical activity guide to healthy active living. Ottawa: Health Canada; 1998.

27. Borg G. Psychophysical bases of perceived exertion. Med Sci Sports Exerc 1982;14 $377-87$.

28. Noble BJ, Borg GA, Jacobs I, et al. A category-ratio perceived exertion scale: relationship to blood and muscle lactates and heart rate. Med Sci Sports Exerc 1983;15: 523-8.

29. American College of Sports Medicine. Resource manual for guidelines for exercise testing and prescription. 2nd ed. Philadelphia: Lea \& Febiger; 1993.

3o. $\mathrm{Pu} \mathrm{CT}$, Nelson ME. Aging, function, and exercise. In: Frontera WR, Dawson DM, Slovik DM, editors. Exercise in rehabilitation medicine. Champaign (IL): Human Kinetics; I999. p. 39I-424.

3I. Jakicic JM, Otto AD. Physical activity considerations for the treatment and prevention of obesity. Am J Clin Nutr 2005;82:226S-9S.

32. Jakicic JM. Exercise in the treatment of obesity. Endocrinol Metab Clin North Am 2003;32:967-80.

33. Hambrecht R, Niebauer J, Marburger C, et al. Various intensities of leisure time physical activity in patients with coronary artery disease: effects on cardiorespiratory fitness and progression of coronary atherosclerotic lesions. J Am Coll Cardiol I993;22:468-77.

34. Franklin BA, Swain DP, Shephard RJ. New insights in the prescription of exercise for coronary patients. J Cardiovasc Nurs 2003;18:1r6-23.

35. Warburton DER, Mathur S. Skeletal muscle training in people with chronic heart failure or chronic obstructive pulmonary disease. Physiother Can 2004;56:I43-57.

36. Meyer K, Samek L, Schwaibold M, et al. Interval training in patients with severe chronic heart failure: analysis and recommendations for exercise procedures. Med Sci Sports Exerc I997;29:306-I2.

37. Freedson PS, Miller K. Objective monitoring of physical activity using motion sensors and heart rate. Res Q Exerc Sport 2000;7I:S2I-9.

38. Sirard JR, Pate RR. Physical activity assessment in children and adolescents. Sports Med 200I;3I:439-54.

39. Ainslie P, Reilly T, Westerterp K. Estimating human energy expenditure: a review of techniques with particular reference to doubly labelled water. Sports Med 2003; 33:683-98.

40. Tudor-Locke C, Bassett DR Jr. How many steps/day are enough? Preliminary pedometer indices for public health. Sports Med 2004;34:I-8.

4I. Chan CB, Ryan DA, Tudor-Locke C. Health benefits of a pedometer-based physical activity intervention in sedentary workers. Prev Med 2004;39:1215-22.

42. Tudor-Locke C, Williams JE, Reis JP, et al. Utility of pedometers for assessing physical activity: convergent validity. Sports Med 2002;32:795-808.

43. Tudor-Locke C, Williams JE, Reis JP, et al. Utility of pedometers for assessing physical activity: construct validity. Sports Med 2004;34:28I-9I.

44. Bassett DR Jr, Ainsworth BE, Leggett SR, et al. Accuracy of five electronic pedometers for measuring distance walked. Med Sci Sports Exerc 1996;28:107I-7.

45. Le Masurier GC, Lee SM, Tudor-Locke C. Motion sensor accuracy under controlled and free-living conditions. Med Sci Sports Exerc 2004;36:905-Io.

46. Schneider PL, Crouter SE, Bassett DR. Pedometer measures of free-living physical activity: comparison of I3 models. Med Sci Sports Exerc 2004;36:33I-5.

47. Le Masurier GC, Tudor-Locke C. Comparison of pedometer and accelerometer accuracy under controlled conditions. Med Sci Sports Exerc 2003;35:867-71.

Correspondence to: Dr. Darren E.R. Warburton, Cardiovascular Physiology and Rehabilitation Laboratory, University of British Columbia, Unit II, Osborne Centre, 6ro8 Thunderbird Blvd., Vancouver BC V6T IZ3; fax 604 822-945I; darren.warburton@ubc.ca 


\section{REVIEW}

\section{Appendix 1: The Healthy Physical Activity Participation Questionnaire}

\section{A. Answer the following questions}

\section{Frequency}

Over a typical 7-day period ( 1 week), how many times do you engage in physical activity that is sufficiently prolonged and intense to cause sweating and a rapid heart beat?

$\square$ At least three times

$\square$ Normally once or twice

$\square$ Rarely or never

Intensity

When you engage in physical activity, do you have the impression that you:

$\square$ Make an intense effort

$\square$ Make a moderate effort

$\square$ Make a light effort

Perceived fitness

In a general fashion, would you say that your current physical fitness is:
$\square$ Very good
$\square$ Good
$\square$ Average
$\square$ Poor
$\square$ Very poor

B. Circle your score below for each answer and total your score

\begin{tabular}{|c|c|c|c|c|c|c|}
\hline Item & Male & Female & Male & Female & Male & Female \\
\hline \multirow[t]{2}{*}{ Frequency } & \multicolumn{2}{|c|}{ Rarely or never } & \multicolumn{2}{|c|}{ Normally once or twice } & \multicolumn{2}{|c|}{ At least 3 times } \\
\hline & 0 & 0 & 2 & 3 & 3 & 5 \\
\hline \multirow[t]{2}{*}{ Intensity } & \multicolumn{2}{|c|}{ Light effort } & \multicolumn{2}{|c|}{ Moderate effort } & \multicolumn{2}{|c|}{ Intense effort } \\
\hline & 0 & 0 & 1 & 2 & 3 & 3 \\
\hline \multirow[t]{2}{*}{ Perceived fitness } & \multicolumn{2}{|c|}{ Very poor or poor } & \multicolumn{2}{|c|}{ Average } & \multicolumn{2}{|c|}{ Good or very good } \\
\hline & 0 & 0 & 3 & 1 & 5 & 3 \\
\hline
\end{tabular}

C. Determine the health benefits of your physical activity based on your total score

$\begin{array}{cl}\text { Total score } & \text { Health benefit } \\ 9-11 & \text { Excellent } \\ 6-8 & \text { Very good } \\ 4-5 & \text { Good } \\ 1-3 & \text { Fair } \\ 0 & \text { Needs improvement }\end{array}$

Source: Canadian Physical Activity, Fitness and Lifestyle Approach. ${ }^{2}$ 